\title{
Folding and Assembly of Proteorhodopsin
}

\author{
Adriana L. Klyszejko ${ }^{1} \dagger$, Sarika Shastri ${ }^{2} \dagger$, Stefania A. Mari ${ }^{3}$, \\ Helmut Grubmüller ${ }^{4}$, Daniel J. Muller ${ }^{1 *}$ and Clemens Glaubitz ${ }^{2}$
}

\author{
${ }^{1}$ Biotechnology Center \\ University of Technology \\ Dresden, Germany \\ ${ }^{2}$ Institute of Biophysical \\ Chemistry and Center for \\ Biomolecular Magnetic \\ Resonance, Goethe University \\ Frankfurt, Germany \\ ${ }^{3}$ Institute of General \\ Physiology and Biological \\ Chemistry, University \\ of Milan, Italy \\ ${ }^{4}$ Max-Planck-Institute of \\ Biophysical Chemistry, \\ Göttingen, Germany
}

Received 5 October 2007; accepted 9 November 2007

Available online

19 November 2007

Edited by W. Baumeister
Proteorhodopsins (PRs), the recently discovered light-driven proton pumps, play a major role in supplying energy for microbial organisms of oceans. In contrast to PR, rhodopsins found in Archaea and Eukarya are structurally well characterized. Using single-molecule microscopy and spectroscopy, we observed the oligomeric assembly of native PR molecules and detected their folding in the membrane. PR showed unfolding patterns identical with those of bacteriorhodopsin and halorhodopsin, indicating that PR folds similarly to archaeal rhodopsins. Surprisingly, PR predominantly assembles into hexameric oligomers, with a smaller fraction assembling into pentamers. Within these oligomers, PR arranged into radial assemblies. We suggest that this structural assembly of PR may have functional implications.

(C) 2007 Elsevier Ltd. All rights reserved.

\section{Introduction}

Rhodopsins occur in Archaea, Bacteria, and Eukarya. Using a chromophore, rhodopsins sense light of certain wavelengths. In Archaea, this captured light is employed to pump protons or chloride ions across the membrane and to create an electrochemical gradient, which powers cellular machineries. Other archaeal rhodopsins sense light for phototaxis. ${ }^{1}$ In vertebrates, rhodopsins function primarily as sensory proteins and account for vision. ${ }^{2}$ Archaeal and eukaryal rhodopsins show common structural motifs; they are embedded in the membrane bilayer and have seven transmem-

*Corresponding author. E-mail address:

mueller@biotec.tu-dresden.de.

$\uparrow$ A.K. and S.S. contributed equally to this work.

Abbreviations used: AFM, atomic force microscopy; PR, proteorhodopsin; SMFS, single-molecule force spectroscopy; F-D, force-distance; DOPC, 1,2-dioleolyl-sn-glycero-3-phosphocholine. brane $\alpha$-helices surrounding the light-sensitive retinal. However, the oligomeric assembly of rhodopsins in Archaea and Eukarya differs and ranges from monomeric, to dimeric, to trimeric forms, which supposedly have structural and functional origins. 1,

The recent discovery of rhodopsins in bacteria [proteorhodopsin (PR)] came after the sequence analysis of a cloned genome region from a marine bacterium of the uncultivated SAR86 clade. ${ }^{5}$ Subsequent DNA screening of microorganisms from different oceans revealed a very large diversity of PR belonging to divergent clades of the Alphaproteobacteria and Gammaproteobacteria classes. ${ }^{6-11}$ Two related PR families that absorb light with different absorption maxima, $\approx 525 \mathrm{~nm}$ (green) and $\approx 490 \mathrm{~nm}$ (blue), were found, and their distribution was shown to be stratified with water depth. A single amino acid at position 105 functions as a spectral tuning switch and seems to account for most of the spectral difference between green-absorbing PRs and blue-absorbing PRs. ${ }^{10}$ The phototrophy conferred by PR can provide critical amounts of energy 
not only for respiration and maintenance but also for active growth of marine bacterioplankton in their natural environment. ${ }^{12}$ This potential to complement their chemotropic lifestyle by phototrophy has evolutionarily favored these oceanophilic microbial organisms and the wide oceanographic distribution of PR genes. ${ }^{6,13-15}$ PRs overproduced in Escherichia coli have further established that PR functions as a light-driven proton pump with the potential to generate energy for cell growth and maintenance. $5,9,16$

PR is a light-driven proton pump like bacteriorhodopsin from archaeabacteria. ${ }^{1,17}$ In contrast to PR, the structure-function relationship of bacteriorhodopsin and its homologues, the light-driven chloride pump halorhodopsin ${ }^{18}$ and sensory rhodopsin, ${ }^{3}$ has been studied at molecular resolution. So far, the only structural insights available for PR have been revealed from spectroscopic studies and modeling. ${ }^{19,20}$ Recent low-resolution structural data revealed from two-dimensionally crystallized PR suggested a hexagonal lattice constant of $\approx 8.7 \mathrm{~nm}$, ${ }^{21}$ showing a symmetric ringlike arrangement. $^{22}$ Initial solid-state NMR studies focused on the characterization of retinal and Schiff base. ${ }^{23}$ Here, we show high-resolution atomic force microscopy (AFM) topographs of green PR reconstituted into the lipid bilayer. These topographs show oligomers of PRs assembled in a two-dimensional lattice and densely packed in the membrane. Single-molecule force spectroscopy (SMFS) shows PRs to unfold via similar structural intermediates as observed for bacteriorhodopsin and halorhodopsin. This suggests that all three rhodopsins have almost identical folding and three-dimensional structures.

\section{Results and Discussions}

\section{Two-dimensional PR crystals show larger unit cell size than bacteriorhodopsin}

AFM imaging of reconstituted PR membranes in buffer solution (Fig. 1a) showed crystalline $\left(^{*}\right)$ and densely packed ${ }^{* *}$ ) regions. At high resolution, the surface of the two-dimensional PR crystals revealed donut-like structures arranged in a hexagonal lattice of $8.8 \pm 0.7 \mathrm{~nm}$ (mean $\pm \mathrm{SD} ; n=30$ ) side length (Fig. $1 \mathrm{~b})$, which agrees well with the low-resolution electron microscopy analysis of the same crystals. ${ }^{22}$ These donut-like structures featured pronounced protrusions extending $0.9 \pm 0.3 \mathrm{~nm}(n=50)$ above the lipid surface. In comparison, the hexagonal lattice of bacteriorhodopsin trimers in native purple membrane patches shows a side length of $\approx 6.2 \mathrm{~nm}^{24}$ with single bacteriorhodopsin molecules protruding between $\approx 0.5$ and $0.8 \mathrm{~nm}$ from the lipid bilayer. ${ }^{25}$ The hexagonal unit cell of the purple membrane lattice covers an area of $16.64 \mathrm{~nm}^{2}$, hosting three bacteriorhodopsin molecules. In contrast, the area covered by the unit cell of the hexagonal PR lattice is $33.53 \mathrm{~nm}^{2}$. It was suggested that the secondary structures of PR and bacteriorhodopsin are very similar. ${ }^{19}$ Thus, the unit cell of the PR lattice would provide sufficient space to accommodate up to six PR molecules instead of three as observed for bacteriorhodopsin. Recent calculations suggested $\approx 24,000$ PR molecules per SAR86 cell. ${ }^{26}$ Packed into a densely packed crystalline lattice, such as observed for purple membrane, this would make a 600-nm-diameter flat circular patch covering a significant portion of a cell surface. Therefore, the
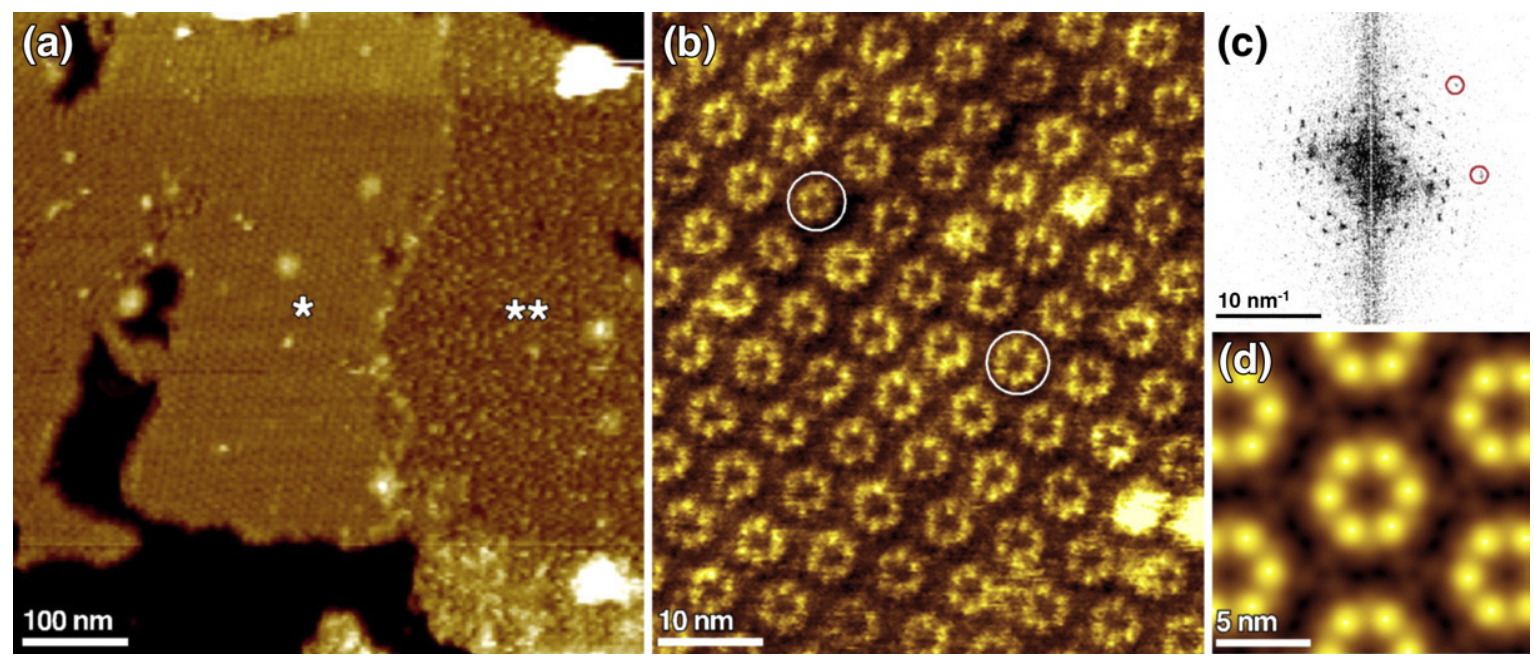

Fig. 1. AFM of two-dimensionally crystallized and densely packed PR membranes. (a) Survey showing crystalline $(*)$ and densely packed $(* *)$ patches of PRs embedded in the lipid (DOPC) bilayer. The lipid bilayer protruded $4.5 \pm 0.5 \mathrm{~nm}$ $(n=10)$ from the support, the crystals protruded $7.1 \pm 0.5 \mathrm{~nm}(n=10)$, and the densely packed PRs protruded $6.3 \pm 0.5 \mathrm{~nm}$ $(n=10)$. (b) High-resolution topography showing the hexagonal lattice $(a=8.8 \pm 0.7 \mathrm{~nm})$ of donut-shaped oligomers formed by PRs. (c) Calculated diffraction pattern of (b) documents spots (red circles), suggesting a lateral resolution of $\approx 1.5 \mathrm{~nm}$. (d) Correlation average of (b). The symmetrized average showed a 9\% deviation from 6-fold symmetry. AFM topographs recorded in buffer solution exhibit a full gray level corresponding to a vertical scale of $20 \mathrm{~nm}(\mathrm{a}) \mathrm{and} 2 \mathrm{~nm}(\mathrm{~b}$ and $\mathrm{d})$. 
half packing density of PR would cover twice the area of the cell membrane. Hence, the enhanced size of the PR donuts leads to the question whether they reflect the oligomeric state of trimers, tetramers, pentamers, or hexamers.

Most of the donut-shaped molecules observed in the high-resolution AFM images (>90\%) of the crystalline PR patches showed the same diameter (Fig. 1b). The donuts showed an average diameter of $4.3 \pm 0.3 \mathrm{~nm}(n=83)$ and, in most cases, six protrusions, which became clearly visible after correlation averaging (Fig. 1d). In contrast, some donut-like shapes exhibited smaller diameters (Fig. 1b, compare circles) and less than six protrusions. However, from these topographs, it could not be unambiguously answered how many PR molecules have established the protrusions.

\section{The oligomeric assembly of PR}

High-resolution AFM of the noncrystalline areas of the reconstituted membrane patches showed densely packed PR oligomers (Fig. 2a). The highest protrusions of these oligomers extended $1.3 \pm 0.2 \mathrm{~nm}$ $(n=50)$ from the surface of the lipid bilayer. The majority of oligomers $(>90 \%)$ observed were circular, with their protrusions being equally distributed at the outer circle diameter. Surprisingly, individual oligomers exhibited either six (Fig. 2b) or five (Fig. 2c) protrusions, thus showing either 6- or 5-fold symmetries. In rare cases, incomplete oligomers missed a single subunit or more (Fig. 2, dotted ellipse). The surface area occupied by a PR molecule $\approx 2 \times 3 \mathrm{~nm}^{2}$ matches that observed for bacteriorhodopsin $^{25}$ and halorhodopsin. ${ }^{27}$ This suggested
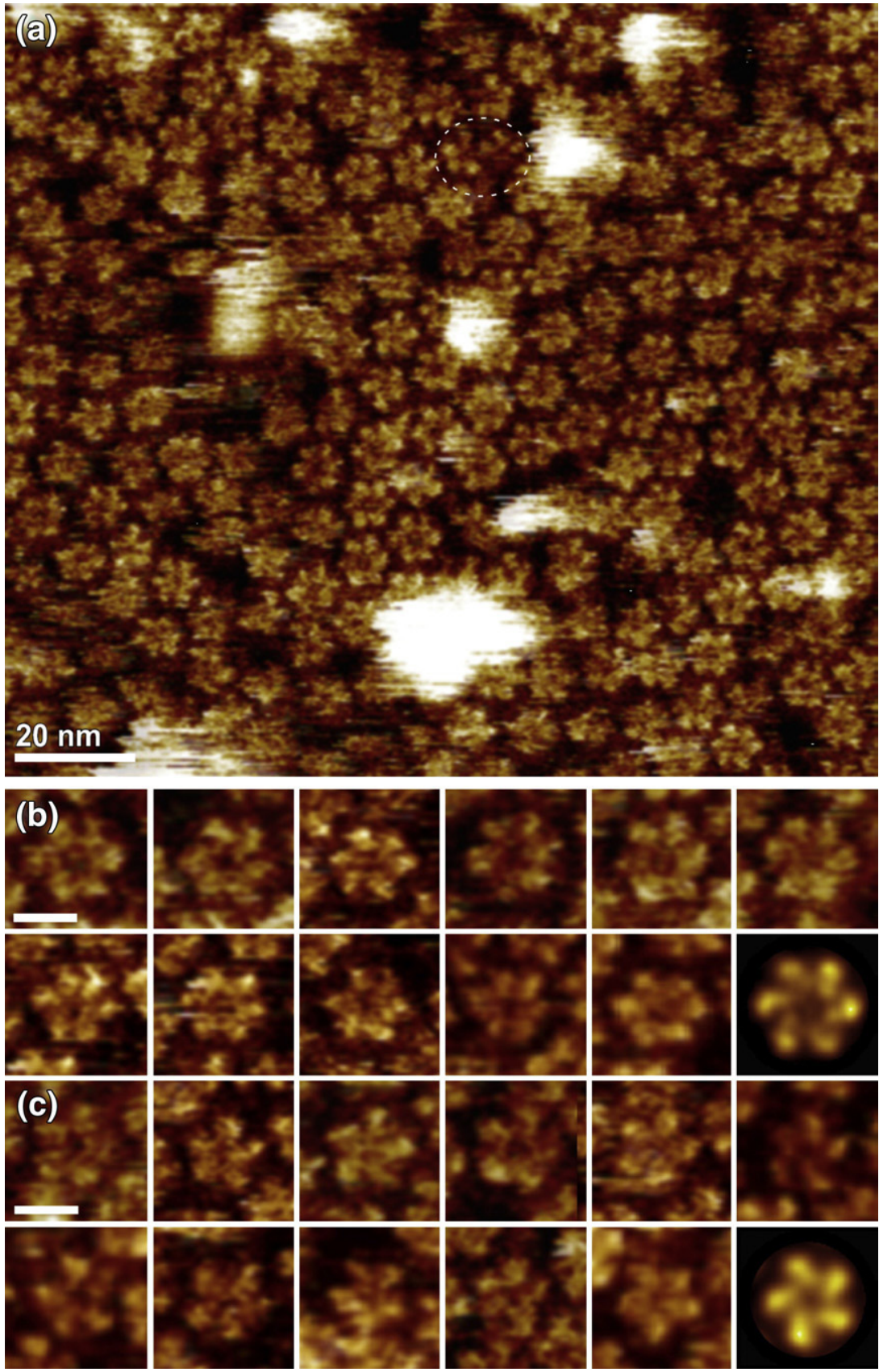

Fig. 2. High-resolution AFM of densely packed PR oligomers. (a) PR molecules could assemble into different oligomeric forms. Galleries show individual hexameric (b) and pentameric (c) PR oligomers. The last oligomers shown (circled black) represent correlation averages. Scale bars in (b) and (c) represent $5 \mathrm{~nm}$. AFM topographs recorded in buffer solution exhibit a full gray level corresponding to a vertical scale of $2 \mathrm{~nm}$. 
that the PR oligomers observed represented either pentamers or hexamers. We further conclude that the hexagonal PR crystals imaged (Fig. 1) predominantly showed hexameric oligomers of PR.

\section{PR, bacteriorhodopsin, and halorhodopsin show similar folding}

After imaging of the crystalline PR patches, the AFM was switched to SMFS. The stylus was then pushed onto the membrane surface, applying a moderate force of $\approx 1 \mathrm{nN}$ for about $1 \mathrm{~s}$. This forced the polypeptide of single PR molecules to adsorb to the AFM stylus. ${ }^{28}$ Upon withdrawal of the stylus, we recorded the AFM cantilever deflection over distance, resulting in force-distance (F-D) curves. ${ }^{28}$ In about $5 \%$ of all cases, the F-D curves showed interaction spectra distributed over a length of $\approx 65-70 \mathrm{~nm}$ (Fig. 3a). We superimposed 30 of these F-D curves (Fig. 3b) to enhance common details among the single-molecule experiments. Previously, identical procedures applied to native bacteriorhodopsin and halorhodopsin ${ }^{27,29}$ showed that the individual force peaks of the F-D spectrum represent the sequential unfolding of single structural segments. ${ }^{28}$ Structural segments such as transmembrane $\alpha$-helices or polypeptide loops establish unfolding intermediates. The SMFS unfolding pattern of PR showed no significant differences to that of bacteriorhodopsin ${ }^{29}$ and halorhodopsin. ${ }^{27}$ This observation strongly suggested that all three light-driven ion pumps fold into very similar secondary structures with seven transmembrane $\alpha$-helices.

\section{How does PR assemble?}

The different oligomeric dimensions of bacteriorhodopsin and PR are clarified when the averaged AFM topographs showing the hexagonal lattice of bacteriorhodopsin trimers from native purple membrane (Fig. 4a) ${ }^{25}$ and of reconstituted PR hexamers (Fig. 4b) are compared. The superimposed outline of bacteriorhodopsin molecules ${ }^{24}$ indicates their tangential assembly into trimers, forming the hexagonal lattice of purple membrane. Singlemolecule unfolding experiments (Fig. 3) together with sequence alignments ${ }^{19}$ suggest that PR, bacteriorhodopsin, and halorhodopsin exhibit similar secondary structures. Thus, we superimposed the outline of bacteriorhodopsin onto the PR hexamer as well. It became clear that if PR shows the same fold as bacteriorhodopsin, the hexameric oligomer could not provide sufficient space to host tangentially assembled PR molecules such as observed for the bacteriorhodopsin trimer. The only way to fit six PR molecules into the hexamer would be to arrange them in a radial manner (Fig. 4). Although the average topographs strongly suggested that all six PR molecules were oriented similarly in relation to the hexamer, the resolution was not sufficient to answer the question whether all six PR molecules pointed
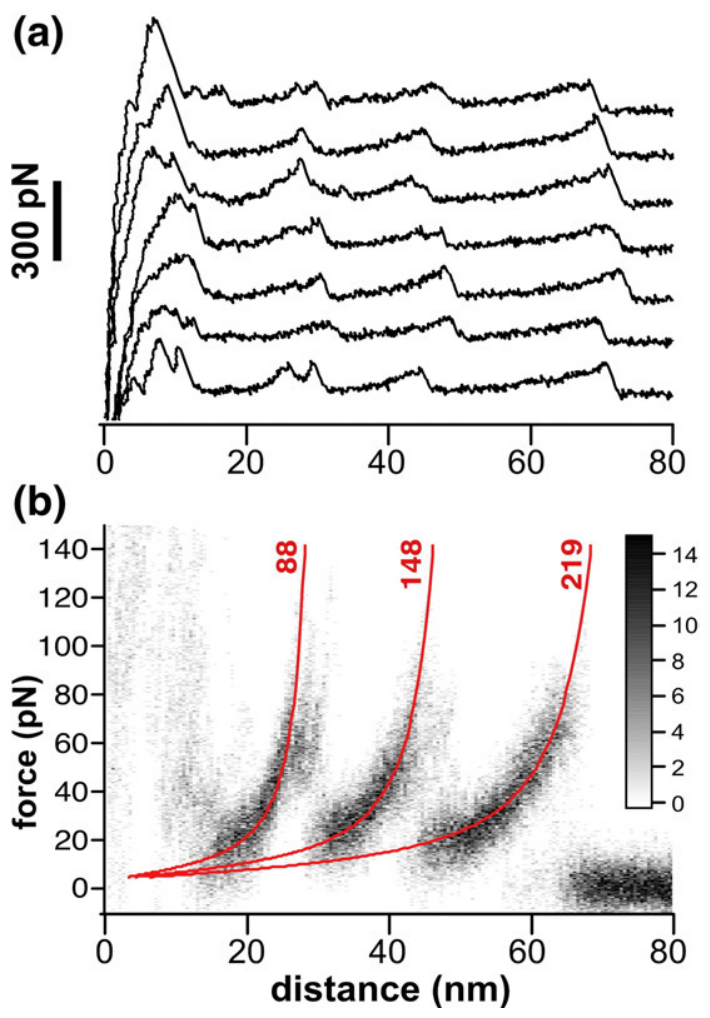

Fig. 3. Unfolding pattern of single PRs embedded within membranes. (a) Individual F-D curves, each one recorded upon unfolding a single PR molecule by SMFS. PR is composed of 249 amino acids. Assuming that the AFM stylus grasps one of the terminal ends and unfolds the protein into a fully stretched conformation reveals an F-D curve exhibiting a maximum length of $\approx 70 \mathrm{~nm}$. (b) Superimposition of $30 \mathrm{~F}-\mathrm{D}$ spectra enhances common patterns. Each force peak detected represents an unfolding intermediate established by structural segments of the PR molecule. Red curves are fits using the worm-like chain model. The three main peaks detected at pulling distances correspond to stretched polypeptide lengths of 88,148 , and 219 amino acids. These distances denoting unfolding intermediates of the PR structure match those measured for bacteriorhodopsin (248 amino acids) and halorhodopsin. ${ }^{27-30}$ SMFS was conducted in buffer solution $(150 \mathrm{mM} \mathrm{KCl}$ and $20 \mathrm{mM}$ Tris- $\mathrm{HCl}, \mathrm{pH} 7.8)$ at a pulling velocity of $87 \mathrm{~nm} / \mathrm{s}$.

toward the inside or the outside of the hexameric center (Fig. 4).

\section{Discussion}

With increasing depth of the ocean, the light available to activate PR becomes rapidly depleted. To convert as much light as possible into a transmembrane proton gradient, planktonic bacteria express a high number $(\approx 24,000)$ of PR molecules. ${ }^{26}$ This proton gradient has been shown to be sufficient to power the bacteria. ${ }^{12,16}$ Light-adapted PR contains about $40 \%$ 13-cis and $60 \%$ all-trans-retinal, a ratio that is shifted to almost exclusive all-trans in darkadapted PR. ${ }^{19,23,31,32}$ Both ratios and the relatively 

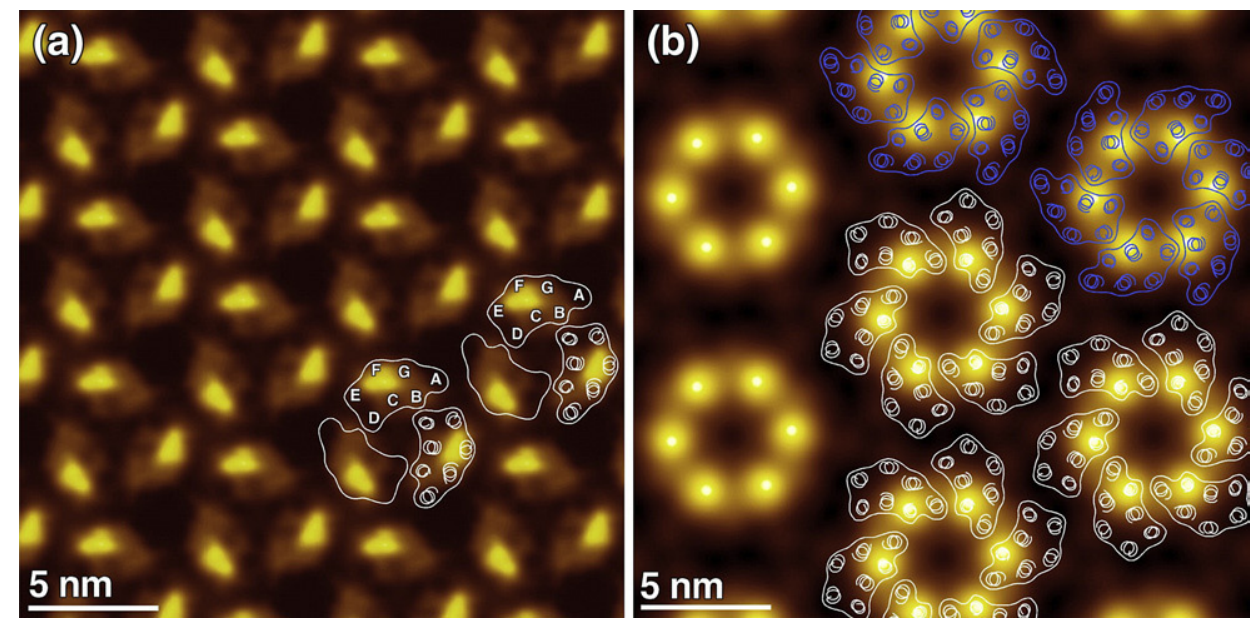

Fig. 4. Correlation-averaged AFM topographs showing the hexagonal assembly of bacteriorhodopsin trimers of native purple membrane (a) and of reconstituted PR hexamers (b). (a) Cytoplasmic purple membrane surface. The highest protrusion of each bacteriorhodopsin molecule corresponds to the polypeptide loop connecting transmembrane $\alpha$-helices $\mathrm{E}$ and $\mathrm{F}^{25}$ Outlined bacteriorhodopsin shapes were adapted from sections close to the cytoplasmic surface of BR trimers obtained from electron crystallographic analyses. ${ }^{24}$ (b) Crystallized PR hexamers. Assuming that each of the six protrusions represents one PR molecule, the proton pumps must be assembled radially to fit into the oligomer. Outlines in white and blue show two out of many possible orientations of the PR molecules. Averaged topographs exhibit a full gray level corresponding to a vertical scale of $2 \mathrm{~nm}$.

slow photocycle of $\mathrm{PR}^{33}$ support the finding that a high number of PR molecules are required to convert sufficient amounts of solar energy to power the bacteria. Such a high number of PR molecules must be packed densely to prevent them from consuming too much space within the cellular membrane. This includes that PR assembles into oligomers. In the case of bacteriorhodopsin and rhodopsin, it has been shown that their oligomeric assembly enhances their structural stability.1,4,34 Because intramembranous contacts between membrane proteins and their exposure to varying lipid compositions may modulate their function, the homo-oligomerization provides PRs a stable environment and ensures their continuous functionality over an extended range. ${ }^{35}$ In contrast to bacteriorhodopsin and rhodopsin, we have observed the oligomeric state of PR being reconstituted into a lipid bilayer and not in the native cell membrane. However, the reconstitution of PR into different lipids under many different conditions resulted almost every time in the formation of twodimensional crystals such as observed here. ${ }^{22}$ Thus, it is safe to assume that the tendency to assemble into hexamers is intrinsic to the PR structure.

The AFM topographs show that all PRs within the oligomer exposed identical surface features and thus showed similar orientations relative to the membrane plane. Moreover, all PR molecules within the donut-shaped oligomer must be somehow assembled into a radial symmetric arrangement. We could not resolve whether the PR molecules point with their chromophore inside or outward to the oligomeric center. Spectroscopic characterization of the PR photocycle, NMR spectroscopy, and homology modeling suggested that the retinal is oriented and interacts differently with PR compared to bacteriorhodopsin and sensory rhodopsin
II. ${ }^{19,20,23,33}$ Although we do not know yet the exact orientation of the retinal with respect to the cell membrane, the radial distribution of PR around one center may also include functional requirements. As light in the sea is polarized, the radial distribution of chromophores may enhance the probability of one out of six differently oriented PR to absorb light and to contribute to the life-supporting proton gradient. Assuming a homogeneous distribution of such hexameric PRs within the cell membrane, the average light absorption would be independent of the orientation of the bacterial cell to the incident polarized light and a proton gradient would be homogeneously generated over the cell membrane.

\section{Materials and Methods}

\section{Materials}

All-trans retinal was obtained from Sigma-Aldrich, unlabeled amino acids and nucleotide bases for the defined medium were from AppliChem, LB medium was from Roth, and 1,2-dioleolyl-sn-glycero-3-phosphocholine (DOPC) was from Avanti Polar Lipids. $n$-Dodecyl$\beta$-D-maltoside was obtained from AppliChem, $n$-octyl- $\beta$ D-glucopyranoside was from GLYCON Biochemicals $\mathrm{GmbH}$, Triton X-100 was from AppliChem, and Ni-NTA agarose was from Qiagen.

\section{Expression and purification of PR}

Expression of wild-type PR was essentially done as described elsewhere. ${ }^{19,23}$ The cells were grown in LB medium with $50 \mu \mathrm{g} / \mathrm{ml}$ kanamycin. The cells were induced at an $\mathrm{OD}_{578}$ of 0.8 with $1 \mathrm{mM}$ IPTG and $0.7 \mathrm{mM}$ of all-trans-retinal (dissolved in ethanol). Overexpression 
was achieved by a further incubation at $37{ }^{\circ} \mathrm{C}$ for $3-4 \mathrm{~h}$ and was visually observed by a pink color change of the cells. The cells were harvested by centrifugation, and cells were broken using a Constant Systems cell disrupter at 1.5-2 kbar. The recovered membrane pellet was solubilized with $1.5 \%$-dodecyl- $\beta$-D-maltoside overnight. Detergent-solubilized PR (supernatant) was obtained by ultracentrifugation and was incubated with Ni-NTA beads for approximately $1 \mathrm{~h}$. After the protein was thoroughly washed, it was finally eluted in $0.2 \%$ Triton $X-100$. Purity of preparation was checked on SDS-PAGE, UV-Vis spectroscopy, and gel filtration.

\section{Reconstitution and crystallization}

DOPC in chloroform was dried in a stream of nitrogen for about $30 \mathrm{~min}$ followed by vacuum drying. Lipid was solubilized in $2 \% n$-octyl- $\beta$-D-glucopyranoside dissolved in equilibration buffer ( $20 \mathrm{mM}$ 4-morpholineethanesulfonic acid and $300 \mathrm{mM} \mathrm{NaCl}, \mathrm{pH}$ 6.0) at a concentration of $5 \mathrm{mg} / \mathrm{ml}$ and sonicated until clear. PR was reconstituted at a very low lipid-to-protein ratio of $\sim 0.25(\mathrm{w} / \mathrm{w})$. Typical crystalline preparations ${ }^{22}$ involved slow detergent removal by dialysis with the aid of dialysis tubes $(14 \mathrm{kDa}$ cutoff) against excess of dialysis buffer (50 mM Tricin, $100 \mathrm{mM} \mathrm{NaCl}, 10 \mathrm{mM} \mathrm{MgCl}_{2}, 3 \mathrm{mM} \mathrm{NaN}_{3}, 5 \mathrm{mM}$ DTT, $\mathrm{pH} 8.5$, and 7.5\% methyl-2,4-pentanediol). The dialysis buffer was changed every day, and the crystalline samples were obtained after 7 days. These samples were then used for measurements.

\section{High-resolution AFM imaging}

Reconstituted PR membranes were adsorbed onto freshly cleaved mica in buffer solution $(300 \mathrm{mM} \mathrm{KCl}$, and $20 \mathrm{mM}$ Tris- $\mathrm{HCl}, \mathrm{pH}$ 8.0). ${ }^{36}$ After this, the sample was washed with imaging buffer $(150 \mathrm{mM} \mathrm{KCl}, 20 \mathrm{mM}$ $\mathrm{MgCl}_{2}$, and $20 \mathrm{mM}$ Tris- $\mathrm{HCl}, \mathrm{pH}$ 8.5) to remove weakly attached membranes. The AFM used was a Nanoscope III (Digital Instruments, Santa Barbara, CA) equipped with a J-scanner $(\approx 100 \mu \mathrm{m})$ and an oxide-sharpened $\mathrm{Si}_{3} \mathrm{~N}_{4}$ stylus on a cantilever with a nominal spring constant of $\approx 0.1 \mathrm{~N} / \mathrm{m}$ (OMCL TR-400-PS, Olympus, Tokyo, Japan). Contact mode AFM imaging was performed by electrostatically damping the forces applied locally between the AFM stylus and protein membrane to $\approx 50 \mathrm{pN}^{37}$ Such low forces prevented possible deformations of the protein by the scanning AFM stylus. ${ }^{25}$ Topographs recorded in trace and retrace scanning directions showed no significant differences, confirming that the protein surface was not disturbed by the scanning process of the AFM. All measurements were carried out at room temperature.

\section{SMFS}

The protein membrane was first imaged by AFM to mechanically unfold individual PRs using SMFS. Then, the AFM stylus was brought into contact with the membrane protein surface. Applying a constant force of $\approx 1-1.5 \mathrm{nN}$ for $\approx 1 \mathrm{~s}$ ensured that one terminal end of the protein attached to the stylus via nonspecific interactions. ${ }^{27,28,30}$ Separation of the stylus and membrane stretched this molecular bridge and exerted a force at the protein, leading to its unfolding. The F-D spectra recorded during the unfolding process showed characteristic patterns similar to those previously assigned to the unfolding of one bacteriorhodopsin molecule ${ }^{29,30}$ or to the unfolding of one halorhodopsin molecule ${ }^{27}$ by pulling from the C-terminal end. The first $20 \mathrm{~nm}$ of the F-D traces exhibited higher noise compared to the remaining trace due to nonspecific AFM stylus-sample interactions. ${ }^{29,30}$ Thus, force peaks lying within this region are masked by noise and show higher deviations compared to peaks detected at separations above $20 \mathrm{~nm}$.

\section{Acknowledgements}

We thank C. Bippes, D. Cisneros, A. Kedrov, T. Sapra, D. Oesterhelt, and K. Palczewski for their help. J. Vonck and W. Kühlbrandt are acknowledged for their help in setting up the 2D crystallization. This work was supported by the European Union, the Deutsche Forschungsgemeinschaft, and the SFB 472 (Molecular Bioenergetics).

\section{References}

1. Oesterhelt, D. (1998). The structure and mechanism of the family of retinal proteins from halophilic archaea. Curr. Opin. Struct. Biol. 8, 489-500.

2. Palczewski, K. (2006). G protein-coupled receptor rhodopsin. Annu. Rev. Biochem. 75, 743-767.

3. Luecke, H., Schobert, B., Lanyi, J. K., Spudich, E. N. \& Spudich, J. L. (2001). Crystal structure of sensory rhodopsin II at 2.4 angstroms: insights into color tuning and transducer interaction. Science, 293, 1499-1503.

4. Fotiadis, D., Jastrzebska, B., Philippsen, A., Muller, D. J., Palczewski, K. \& Engel, A. (2006). Structure of the rhodopsin dimer: a working model for G-protein-coupled receptors. Curr. Opin. Struct. Biol. 16, 252-259.

5. Beja, O., Aravind, L., Koonin, E. V., Suzuki, M. T., Hadd, A., Nguyen, L. P. et al. (2000). Bacterial rhodopsin: evidence for a new type of phototrophy in the sea. Science, 289, 1902-1906.

6. de la Torre, J. R., Christianson, L. M., Beja, O., Suzuki, M. T., Karl, D. M., Heidelberg, J. \& DeLong, E. F. (2003). Proteorhodopsin genes are distributed among divergent marine bacterial taxa. Proc. Natl Acad. Sci. USA, 100, 12830-12835.

7. Sabehi, G., Massana, R., Bielawski, J. P., Rosenberg, M., Delong, E. F. \& Beja, O. (2003). Novel proteorhodopsin variants from the Mediterranean and Red Seas. Environ. Microbiol. 5, 842-849.

8. Venter, J. C., Remington, K., Heidelberg, J. F., Halpern, A. L., Rusch, D., Eisen, J. A. et al. (2004). Environmental genome shotgun sequencing of the Sargasso Sea. Science, 304, 66-74.

9. Giovannoni, S. J., Bibbs, L., Cho, J. C., Stapels, M. D., Desiderio, R., Vergin, K. L. et al. (2005). Proteorhodopsin in the ubiquitous marine bacterium SAR11. Nature, 438, 82-85.

10. Man, D., Wang, W., Sabehi, G., Aravind, L., Post, A. F., Massana, R. et al. (2003). Diversification and spectral tuning in marine proteorhodopsins. $E M B O \mathrm{~J} . \mathbf{2 2}$, 1725-1731.

11. Sabehi, G., Loy, A., Jung, K. H., Partha, R., Spudich, J. L., Isaacson, T. et al. (2005). New insights into 
metabolic properties of marine bacteria encoding proteorhodopsins. PLoS Biol. 3, e273.

12. Gomez-Consarnau, L., Gonzalez, J. M., Coll-Llado, M., Gourdon, P., Pascher, T., Neutze, R. et al. (2007) Light stimulates growth of proteorhodopsin-containing marine Flavobacteria. Nature, 445, 210-213.

13. Bielawski, J. P., Dunn, K. A., Sabehi, G. \& Beja, O (2004). Darwinian adaptation of proteorhodopsin to different light intensities in the marine environment. Proc. Natl Acad. Sci. USA, 101, 14824-14829.

14. Sabehi, G., Beja, O., Suzuki, M. T., Preston, C. M. \& DeLong, E. F. (2004). Different SAR86 subgroups harbour divergent proteorhodopsins. Environ. Microbiol. 6, 903-910.

15. Frigaard, N. U., Martinez, A., Mincer, T. J. \& DeLong, E. F. (2006). Proteorhodopsin lateral gene transfer between marine planktonic Bacteria and Archaea. Nature, 439, 847-850.

16. Walter, J. M., Greenfield, D., Bustamante, C. \& Liphardt, J. (2007). Light-powering Escherichia coli with proteorhodopsin. Proc. Natl Acad. Sci. USA, 104, 2408-2412.

17. Oesterhelt, D. \& Stoeckenius, W. (1973). Functions of a new photoreceptor membrane. Proc. Natl Acad. Sci. USA, 70, 2853-2857.

18. Kolbe, M., Besir, H., Essen, L. O. \& Oesterhelt, D. (2000). Structure of the light-driven chloride pump halorhodopsin at $1.8 \AA$ A resolution. Science, 288, 1390-1396.

19. Friedrich, T., Geibel, S., Kalmbach, R., Chizhov, I., Ataka, K., Heberle, J. et al. (2002). Proteorhodopsin is a light-driven proton pump with variable vectoriality. J. Mol. Biol. 321, 821-838.

20. Hillebrecht, J. R., Galan, J., Rangarajan, R., Ramos, L., McCleary, K., Ward, D. E. et al. (2006). Structure, function, and wavelength selection in blue-absorbing proteorhodopsin. Biochemistry, 45, 1579-1590.

21. Liang, H., Whited, G., Nguyen, C. \& Stucky, G. D. (2007). The directed cooperative assembly of proteorhodopsin into 2D and 3D polarized arrays. Proc. Natl Acad. Sci. USA, 104, 8212-8217.

22. Shastri, S., Vonck, J., Pfleger, N., Haase, W., Kuehlbrandt, W. \& Glaubitz, C. (2007). Proteorhodopsin: characterisation of $2 \mathrm{D}$ crystals by electron microscopy and solid state NMR.BBA Biomembranes, in press, doi:10.1096/10.1016/j.bbamem.2007.10.001.

23. Pfleger, N., Lorch, M., Woerner, A., Shastri, S. \& Glaubitz, C. (2007). Characterisation of Schiff base and chromophore in green proteorhodopsin by solid-state NMR. J. Biomol. NMR, in press, doi:10.1007/s10858007-9203-5.

24. Grigorieff, N., Ceska, T. A., Downing, K. H., Baldwin, J. M. \& Henderson, R. (1996). Electron-crystallo- graphic refinement of the structure of bacteriorhodopsin. J. Mol. Biol. 259, 393-421.

25. Müller, D. J., Sass, H.-J., Müller, S., Büldt, G. \& Engel, A. (1999). Surface structures of native bacteriorhodopsin depend on the molecular packing arrangement in the membrane. J. Mol. Biol. 285, 1903-1909.

26. Beja, O., Spudich, E. N., Spudich, J. L., Leclerc, M. \& DeLong, E. F. (2001). Proteorhodopsin phototrophy in the ocean. Nature, 411, 786-789.

27. Cisneros, D. A., Oesterhelt, D. \& Muller, D. J. (2005). Probing origins of molecular interactions stabilizing the membrane proteins halorhodopsin and bacteriorhodopsin. Structure, 13, 235-242.

28. Kedrov, A., Janovjak, H., Sapra, K. T. \& Muller, D. J. (2007). Deciphering molecular interactions of native membrane proteins by single-molecule force spectroscopy. Annu. Rev. Biophys. Biomol. Struct. 36, 233-260.

29. Müller, D. J., Kessler, M., Oesterhelt, F., Moeller, C., Oesterhelt, D. \& Gaub, H. (2002). Stability of bacteriorhodopsin alpha-helices and loops analyzed by single-molecule force spectroscopy. Biophys. J. 83, 3578-3588.

30. Oesterhelt, F., Oesterhelt, D., Pfeiffer, M., Engel, A., Gaub, H. E. \& Müller, D. J. (2000). Unfolding pathways of individual bacteriorhodopsins. Science, 288, 143-146.

31. Dioumaev, A. K., Brown, L. S., Shih, J., Spudich, E. N., Spudich, J. L. \& Lanyi, J. K. (2002). Proton transfers in the photochemical reaction cycle of proteorhodopsin. Biochemistry, 41, 5348-5358.

32. Imasheva, E. S., Shimono, K., Balashov, S. P., Wang, J. M., Zadok, U., Sheves, M. et al. (2005). Formation of a long-lived photoproduct with a deprotonated Schiff base in proteorhodopsin, and its enhancement by mutation of Asp227. Biochemistry, 44, 10828-10838.

33. Huber, R., Kohler, T., Lenz, M. O., Bamberg, E., Kalmbach, R., Engelhard, M. \& Wachtveitl, J. (2005). $\mathrm{pH}$-dependent photoisomerization of retinal in proteorhodopsin. Biochemistry, 44, 1800-1806.

34. Sapra, K. T., Besir, H., Oesterhelt, D. \& Muller, D. J. (2006). Characterizing molecular interactions in different bacteriorhodopsin assemblies by single-molecule force spectroscopy. J. Mol. Biol. 355, 640-650.

35. Engelman, D. M. (2005). Membranes are more mosaic than fluid. Nature, 438, 578-580.

36. Müller, D. J., Amrein, M. \& Engel, A. (1997). Adsorption of biological molecules to a solid support for scanning probe microscopy. J. Struct. Biol. 119, 172-188.

37. Müller, D. J., Fotiadis, D., Scheuring, S., Müller, S. A. \& Engel, A. (1999). Electrostatically balanced subnanometer imaging of biological specimens by atomic force microscopy. Biophys. J. 76, 1101-1111. 